\title{
Next-Generation Sequencing as a Tool to Detect Vaginal Microbiota Disturbances during Pregnancy
}

\author{
Agnieszka Sroka-Oleksiak ${ }^{1}$, Tomasz Gosiewski ${ }^{1}$ (i) ${ }^{\text {Wojciech Pabian }}{ }^{2}$, Artur Gurgul ${ }^{3}$ (i), \\ Przemysław Kapusta ${ }^{4}$, Agnieszka H. Ludwig-Słomczyńska ${ }^{4}\left(\mathbb{D}\right.$, Paweł P. Wołkow ${ }^{4}(\mathbb{D}$ \\ and Monika Brzychczy-Włoch ${ }^{1, *}$ \\ 1 Department of Molecular Medical Microbiology, Chair of Microbiology, Faculty of Medicine, \\ Jagiellonian University Medical College, 31-121 Krakow, Poland; agnieszka.sroka@uj.edu.pl (A.S.-O.); \\ tomasz.gosiewski@uj.edu.pl (T.G.) \\ 2 Clinical Department of Gynecological Endocrinology and Gynecology, Jagiellonian University Medical \\ College, Kopernika 23, 31-501 Krakow, Poland; mopabian@cyfronet.pl \\ 3 Center for Experimental and Innovative Medicine, University of Agriculture in Krakow, Redzina 1c, \\ 30-248 Kraków, Poland; artur.gurgul@urk.edu.pl \\ 4 Center for Medical Genomics OMICRON, Jagiellonian University Medical College, Kopernika 7c, \\ 31-034 Krakow, Poland; przemyslaw.kapusta@uj.edu.pl (P.K.); agnieszka.ludwig@uj.edu.pl (A.H.L.-S.); \\ pawel.wolkow@uj.edu.pl (P.P.W.) \\ * Correspondence: m.brzychczy-wloch@uj.edu.pl; Tel.: +48-1263-325-67
}

Received: 28 October 2020; Accepted: 16 November 2020; Published: 18 November 2020

\begin{abstract}
The physiological microbiota of the vagina is responsible for providing a protective barrier, but Some factors can disturb the balance in its composition. At that time, the amounts of the genus Lactobacillus decrease, which may lead to the development of infection and severe complications during pregnancy. The aim of the study was the analysis of the bacterial composition of the vagina in 32 Caucasian women at each trimester of pregnancy using the next-generation sequencing method and primers targeting V3-V4 regions. In the studied group, the dominant species were Lactobacillus iners, Lactobacillus gasseri, and Lactobacillus plantarum. Statistically significant differences in the quantitative composition between trimesters were observed in relation to Lactobacillus jensenii, Streptococcus agalactiae, Lactobacillus iners, Gardnerella spp. Out of the 32 patients, 20 demonstrated fluctuations within the genus Lactobacillus, and 9 of them, at different stages of pregnancy, exhibited the presence of potentially pathogenic microbiota, among others: Streptococcus agalactiae, Gardnerella spp., Atopobium vaginae, and Enterococcus faecalis. The composition of the vaginal microbiota during pregnancy was subject to partial changes over trimesters. Although in one-third of the studied patients, both the qualitative and quantitative composition of microbiota was relatively constant, in the remaining patients, physiological and potentially pathogenic fluctuations were distinguished.
\end{abstract}

Keywords: pregnant women; vaginal microbiota; next-generation sequencing; Lactobacillus spp.; Streptococcus agalactiae; GBS; Gardnerella spp.

\section{Introduction}

The vaginal microbiota is a sustainable ecosystem which plays a crucial role in the prevention of infectious diseases in non-pregnant women as well as during pregnancy (in both maternal and neonatal health) [1,2]. The quantitative and qualitative composition of the bacterial profile in the vagina in most women is dominated by bacteria of the genus Lactobacillus $[3,4]$. The Lactobacillus species are able to produce lactic acid, lowering the vaginal $\mathrm{pH}$, therefore, they create a barrier against pathogen invasion and have been regarded as a hallmark of vaginal health $[5,6]$. Some factors, 
e.g., antibiotics, douching practices, hormonal disorders, hygiene of intimate parts, systemic diseases, sexual activity, and smoking can predispose to changes in the bacterial composition of the vagina, including colonization with pathogenic microorganisms [6,7]. As a result, the abundance of beneficial lactobacilli decreases [8] and the diversity of streptococci [9], yeast-like fungi (mainly Candida) [6], or anaerobic bacteria (e.g., Gardnerella spp., Prevotella, Megasphaera, Finegoldia,) [10] increases, which is a convenient condition for the development of vaginal microbiota disturbances or bacterial vaginosis $[2,4]$.

Although epidemiological data indicate disorders in the vaginal microbiota, for example, the presence of group B streptococci (GBS) found in 10-30\% of healthy women [11], they do not usually manifest symptoms of infection. These inconspicuous and common microorganisms are dangerous, especially for a developing fetus, because they may lead to premature delivery, miscarriage [8], or other postpartum complications, e.g., neonatal pneumonia, meningitis, and septicemia $[3,10]$. Therefore, knowledge about the microbiological state of the vagina in each pregnant woman should be necessary.

Until recently, the studies about the composition of the vaginal ecosystem were based on the use of a microbiological culture and phenotype identification of specific species. Actually, these methods can be considered fragmentary because they provide only limited information on bacterial communities [4]. Moreover, many aerobic and anaerobic bacteria are difficult to culture, or there is a lack of appropriate phenotypic tests to identify them. More accurate identification can be obtained using high throughput analyses, e.g., next-generation sequencing (NGS). This type of research uses partial primer sequences that attach to the highly conserved hypervariable regions of the bacterial 16S rRNA gene [1]. The obtained amplicons are sequenced and then subjected to phylogenetic analysis consisting of assigning a sequence to a specific unit at the selected taxonomic level (e.g., family, genus, or species). Studies on the human microbiome with the application of this method have definitely shown more richness in the microbiota (both aerobic and anaerobic) than those identified using culturing methods [12]. For this reason, the implementation of the NGS seems to be an appropriate method to monitor the changes in the vaginal composition of bacteria during pregnancy and its relationship with susceptibility to infection, the possibility of premature birth, and postpartum complications $[3,6,13,14]$.

The aim of our study was an evaluation of the semi-quantitative and qualitative dynamics of vaginal microbiota of healthy Caucasian women at the beginning of each trimester of pregnancy with the use of next-generation sequencing.

\section{Materials and Methods}

\subsection{Patients}

The study included 32 healthy pregnant women aged 22-35 years old, without clinical genitourinary symptoms of infection. Detailed inclusion and exclusion criteria are shown in Table 1. The consent obtained from the participants was both informed and written. The study has been approved by the Bioethics Committee (No. KBET/47/B/2009—samples collection and No. KBET/1072.6120.51.2017—standardization of molecular diagnostic methods), and the patients tested gave their written consent for participation in the study. Two swabs from the lower vagina (vaginal introitus), were taken from patients during a routine prenatal visit in each trimester of pregnancy. The remaining information about the patients has been described previously in an article in which the samples were used for research based on culture methods [15]. 
Table 1. Inclusion and exclusion criteria used for the recruitment of patients into the study.

\begin{tabular}{|c|c|}
\hline Inclusion Criteria & Exclusion Criteria \\
\hline $\begin{array}{l}\text { - women in the first trimester of pregnancy, aged } 18-40 \text { years } \\
\text { - absence of clinical signs of urogenital infection } \\
\text { - lack of antibiotic or probiotic use for up to } 30 \text { days before } \\
\text { getting pregnant and during pregnancy } \\
\text { - value of } 0-6 \text { at the } 10 \\
\text {-point Nugent score in the first trimester as a confirmation of } \\
\text { physiological flora of the genitourinary tract- written consent } \\
\text { to participate in the study }\end{array}$ & $\begin{array}{l}\text { - pregnant women under the age of } 18 \text { and over } 40 \text { years old } \\
\text { - women with a high-risk pregnancy } \\
\text { - rupture of the membranes } \\
\text { - gestational diabetes } \\
\text { - use of antibiotics for up to } 30 \text { days before becoming pregnant } \\
\text { or during pregnancy } \\
\text { - diagnosis of bacterial vaginosis } \\
\text { - result of } 7-10 \text { at the } 10 \text {-point Nugent score in the first trimester } \\
\text { - clinical symptoms of urinary tract infection } \\
\text { - lack of written consent to participate in the research }\end{array}$ \\
\hline
\end{tabular}

\subsection{Samples}

The vaginal swabs were placed in a non-nutrient Amies transport medium (Eurotubo) and delivered to the Department of Microbiology, Jagiellonian University Medical College, within about $2 \mathrm{~h}$. Each time, the first vaginal swab was used to prepare a smear stained with the Gram method, to evaluate the vaginal flora condition according to the 10-point Nugent score [16]. The second swab was suspended in $1 \mathrm{~mL}$ of $0.9 \% \mathrm{NaCl}$, vortexed for $1 \mathrm{~min}$, and stored at $-70{ }^{\circ} \mathrm{C}$. From the material obtained, the volume of $500 \mu \mathrm{L}$ was used in the previous study for quantitative and qualitative assessment of culture [15]. The remaining $500 \mu \mathrm{L}$ was used for the present research. Bacterial DNA was isolated from the samples using the procedure based on enzymatic and mechanic lysis, described by Gosiewski [17], following the manufacturer's protocol for the Mini genomic DNA isolation kit (A\&A Biotechnology, Gdynia, Poland). Additionally, along with $20 \mu \mathrm{L}$ lysozyme and $10 \mu \mathrm{L}$ lysostaphin, $10 \mu \mathrm{L}$ of mutanolysin was added to the samples during the enzymatic lysis step. The purity and concentration of the isolates obtained were measured with the use of a NanoDrop spectrophotometer (Thermo Scientific, Waltham, MA, USA).

\subsection{Library Preparation}

The DNA extracted was used to carry out PCR amplification (T100 Thermal Cycler, BioRad), with primers targeting the 16S rRNA gene V3 and V4 regions, primer F: TCGTCGGCAGCG TCAGATGTGTATAAGAGACAGCCTACGGGNGGCWGCAG, and primer R: GTCTCGTGGG CTCGGAGATGTGTATAAGAGACAGGACTACHVGGGTATCTAATCC (adapter sequences are highlighted in gray). The sequences of primers, the composition of the reaction mixture, and the program for amplification are shown in Table 2.

Table 2. The composition of the reaction mixtures, the reagents involved, and PCR reaction thermal profiles.

$\left.\begin{array}{lcc|c}\hline \multicolumn{2}{c}{\text { Final Volume: } 25 \mu \mathrm{L}} & \multicolumn{2}{c}{\text { Thermal Profile }} \\ \hline \mathrm{H}_{2} \mathrm{O} & 10.5 \mu \mathrm{L} & 95^{\circ} \mathrm{C}- & 5 \mathrm{~min} \\ \text { Kapa Biosystems (Roche) } & 12.5 \mu \mathrm{L} & 95^{\circ} \mathrm{C}- & 30 \mathrm{~s} \\ \text { Primer 1 (F) (Genomed) } & 0.5 \mu \mathrm{L} & 55^{\circ} \mathrm{C}- & 30 \mathrm{~s} \\ \text { Primer 2 (R) (Genomed) } & 0.5 \mu \mathrm{L} & 72{ }^{\circ} \mathrm{C}- & 30 \mathrm{~s}\end{array}\right\} 30 \times$

The volume of $5 \mu \mathrm{L}$ of each amplicon was subjected to electrophoretic separation on $1.5 \%$ agarose gel (Prona ABO, Gdańsk, Poland) diluted $10 \times$ by TBE buffer (Sigma-Aldrich). The PCR products (size, $\sim 550 \mathrm{bp}$ ) were visualized in the Quantity One (BioRad, Hercules, CA, USA) application in the presence of UV light. Subsequent steps (purifying, samples indexing, samples quantification, and pooling) were prepared with the use of $16 \mathrm{~S}$ Metagenomic sequencing library preparation protocol (Illumina, San Diego, CA, USA) [18]. 


\subsection{Next-Generation Sequencing}

The pooled library, with a $10 \%$ spike-in PhiX control DNA was applied to the cartridge for sequencing. Sequencing was performed using the Reagent Kit V3 (600 cycles) in the MiSeq platform (Illumina) in the Center for Medical Genomics OMICRON, Jagiellonian University Medical College, Krakow, Poland.

\subsection{Bioinformatics Analysis}

Raw sequencing reads were controlled for quality using FastQC software (Babraham Bioinformatics). No significant loss in read quality, and no overrepresented primers or adapters sequences were detected. The reads were further analyzed using BaseSpace (Illumina San Diego, CA, USA) 16S Metagenomics application, which is a high-performance implementation of the Ribosomal Database Project (RDP) Classifier described by Wang Q. et al. [19]. In brief, the analysis included matching the reads to primer sequences, excluding non-target reads, filtering the low quality reads by base-call quality, length, and ambiguity, and merging the paired-end reads. Then, chimeric reads were detected using the UCHIME tool [20] and further assigned to the taxonomic classes using the RDP algorithm based on the Bayesian approach [19]. The RDP classification was made with respect to the RefSeq RDP 16S v3 database [21]. This version of the database contains 14,676 bacterial and 660 archaea full 16S rRNA gene sequences. Counts of reads classified to the specific taxonomic units were used to assess alpha and beta diversity using Microbiome Analyst software [22]. Alpha diversity, expressed in the Shannon index, was assessed for significance using Kruskal-Wallis test. The beta diversity was evaluated using the Shannon index and was subsequently tested for significance using PRMANOVA, and visualized with PCoA. To further analyze differences in amounts of separate taxa in the study groups, DeSeq2 [23] software was used to normalize read counts and to perform differential analysis. Obtained $p$-values were corrected for multiple testing using the false discovery rate (FDR) procedure [24]. Differences in microbiota profile in separate patients among trimesters were tested for statistical significance using Fisher exact test in contingency tables and two-sided $p$-values. Dependencies between microbiota within separate samples among trimesters were analyzed using the Spearman rank correlation coefficient. Differences in other parameters, such as age, $\mathrm{pH}$, and Nugent among groups, were evaluated using ANOVA analysis. The later statistical analyses were performed using JASP software, version 0.11.1 [25]. The values presented in brackets refer to the arithmetic mean and standard deviation. In every case, $p$-values $<0.05$ were considered statistically significant.

\section{Results}

\subsection{Characteristics of the Study Population}

Thirty-two pregnant women were included in the present study. The mean age in the experimental group was $30.2 \pm 3.9$ years. The remaining features were measured in each trimester: Nugent score (the mean value in pregnant women in trimester I was $1.3 \pm 1.4,1.0 \pm 1.3$ in trimester II and $1.6 \pm 1.8$ in trimester III) and $\mathrm{pH}$ (the mean was $4.7 \pm 0.7,4.9 \pm 0.4$ in trimester I and II and $4.8 \pm 0.5$ in trimester III). There was no statistical significance between the three trimesters concerning the above-described features $(p=1.00)$.

\subsection{Metagenomic Sequencing}

The data obtained by sequencing consisted of 10,123,878 reads (19,869 minimum reads per sample, 221,589 maximum, and 114,116 median) with an average number of reads of 115,044 . A phylogenetic summary of the results is presented in Table 3. 
Table 3. A phylogenetic summary of the results obtained.

\begin{tabular}{cccc}
\hline Taxonomic Level & $\begin{array}{c}\text { Percent }{ }^{\mathbf{1}} \text { of Reads } \\
\text { in 1st Trimester }\end{array}$ & $\begin{array}{c}\text { Percent }{ }^{\mathbf{1}} \text { of Reads in } \\
\text { 2nd Trimester }\end{array}$ & $\begin{array}{c}\text { Percent }{ }^{\mathbf{1}} \text { of Reads in } \\
\text { 3rd Trimester }\end{array}$ \\
\hline kingdom & $98.82 \%$ & $98.17 \%$ & $98.32 \%$ \\
phylum & $98.72 \%$ & $98.07 \%$ & $98.19 \%$ \\
class & $98.66 \%$ & $98.01 \%$ & $98.11 \%$ \\
order & $98.58 \%$ & $97.95 \%$ & $98.02 \%$ \\
family & $98.47 \%$ & $97.85 \%$ & $97.89 \%$ \\
genus & $98.11 \%$ & $97.55 \%$ & $97.14 \%$ \\
species & $47.58 \%$ & $45.22 \%$ & $57.09 \%$ \\
\hline
\end{tabular}

${ }^{1}$ Percentage of reads assigned to the appropriate systematic levels.

Alpha diversity, expressed as the Shannon index and beta diversity (PCoA) in subsequent trimesters, was similar and not statistically significant (respectively: $p=0.493$ and $p=0.845$ ), Figure 1 .

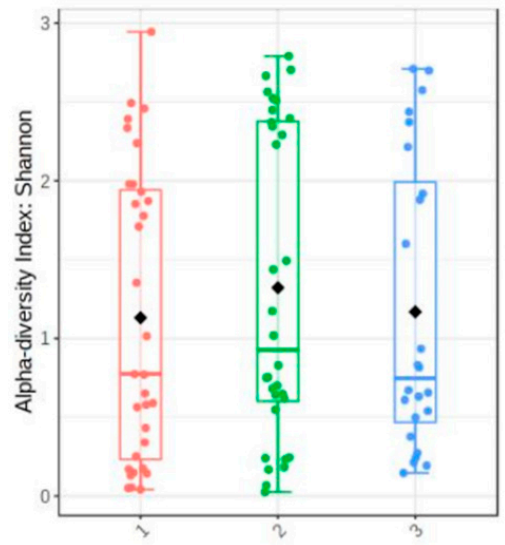

(a)

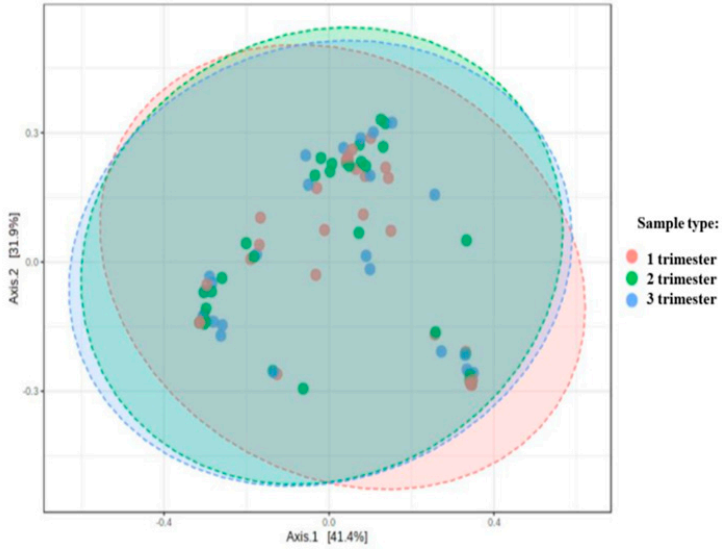

(b)

Figure 1. Alpha and beta diversity expressed as Shannon index (a) and PCoA Jensen-Shannon index (b).

\subsection{Analysis of the Vaginal Microbiota in Each Trimester in the Pregnant Women Group}

Assessment of the vaginal microbiota was conducted at six taxonomic levels (phylum, class, order, family, genus, species). Three of them: L2 (phylum), L6 (genus), and L7 (species) are described in this publication. At the phylum level (L2), all the OTUs obtained were assigned to four phyla: Firmicutes, Actinobacteria, Proteobacteria, and Bacteroidetes. The phylum Firmicutes was clearly predominant in each trimester $(95.43 \%)$, while other phyla constituted negligible amounts $(2.81 \%, 1.27 \%$, and $0.35 \%$, respectively), as shown in Figure 2. The values in parentheses represent the average percentage of bacteria at a specific taxonomic level throughout pregnancy. There were no statistically significant differences between profiles of vaginal microbiota in subsequent trimesters at the phylum level.

At the genus level (L6), the OTUs identified corresponded to 115 genera, but only 5 of them were present in more than 1\% and common to all trimesters: Lactobacillus, Streptococcus; Bifidobacterium, Gardnerella, and Escherichia (Figure 3).

Other genera accounted for less than 1\% (e.g., Prevotella, Staphylococcus, Lactococcus, Bacteroides, Finegoldia, Ureaplasma, Citrobacter, and Faecalibacterium). The abundance of the genus Lactobacillus was $>90 \%$ in 23 pregnant women.

Statistically significant differences were found between 2nd and 3rd trimesters with regard to the abundance of the genus Streptococcus $(p<0.01)$ and also between all trimesters in relation to the genus Gardnerella $(p<0.01)$. 


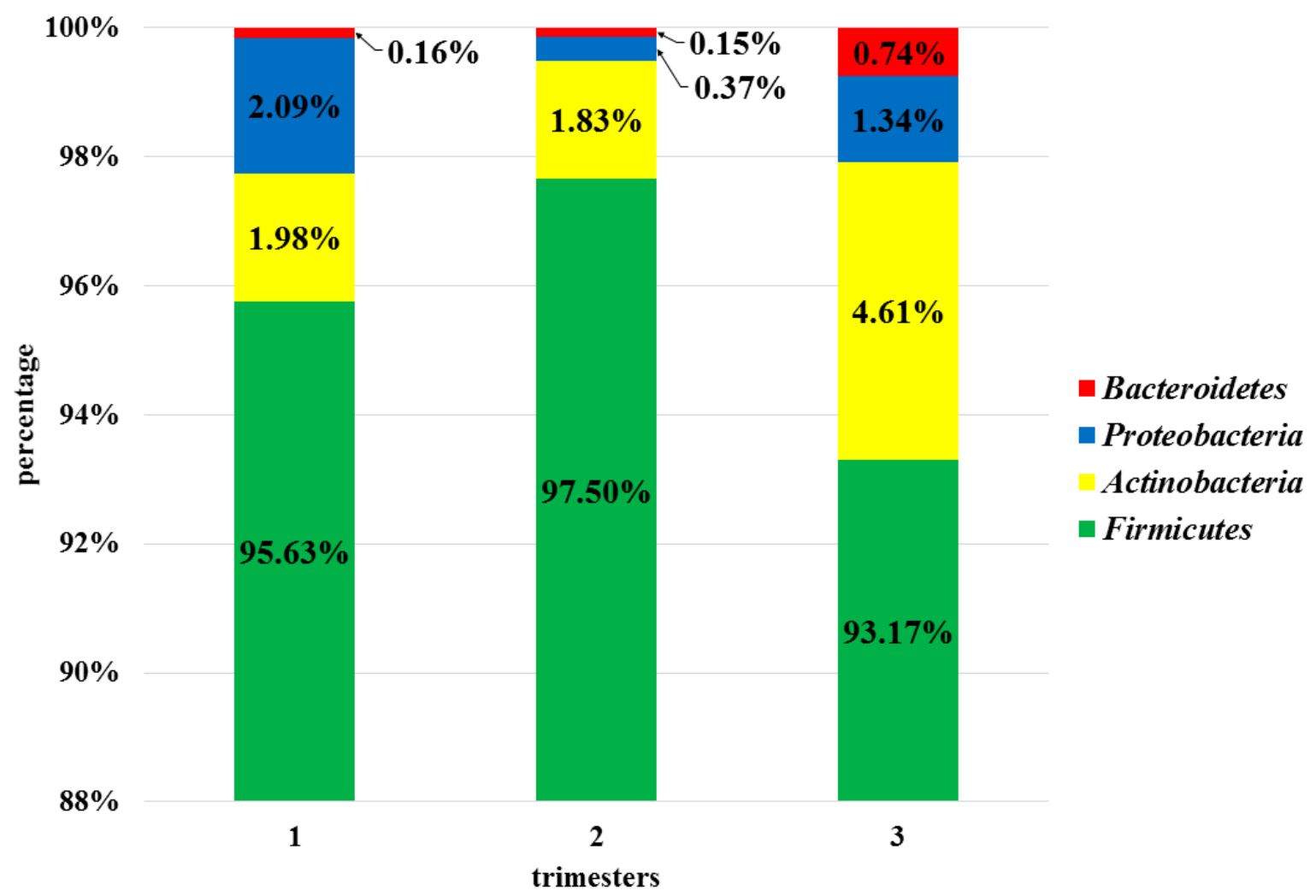

Figure 2. Bacterial profiles for 1st, 2nd, and 3rd trimesters at the phylum level (L2).

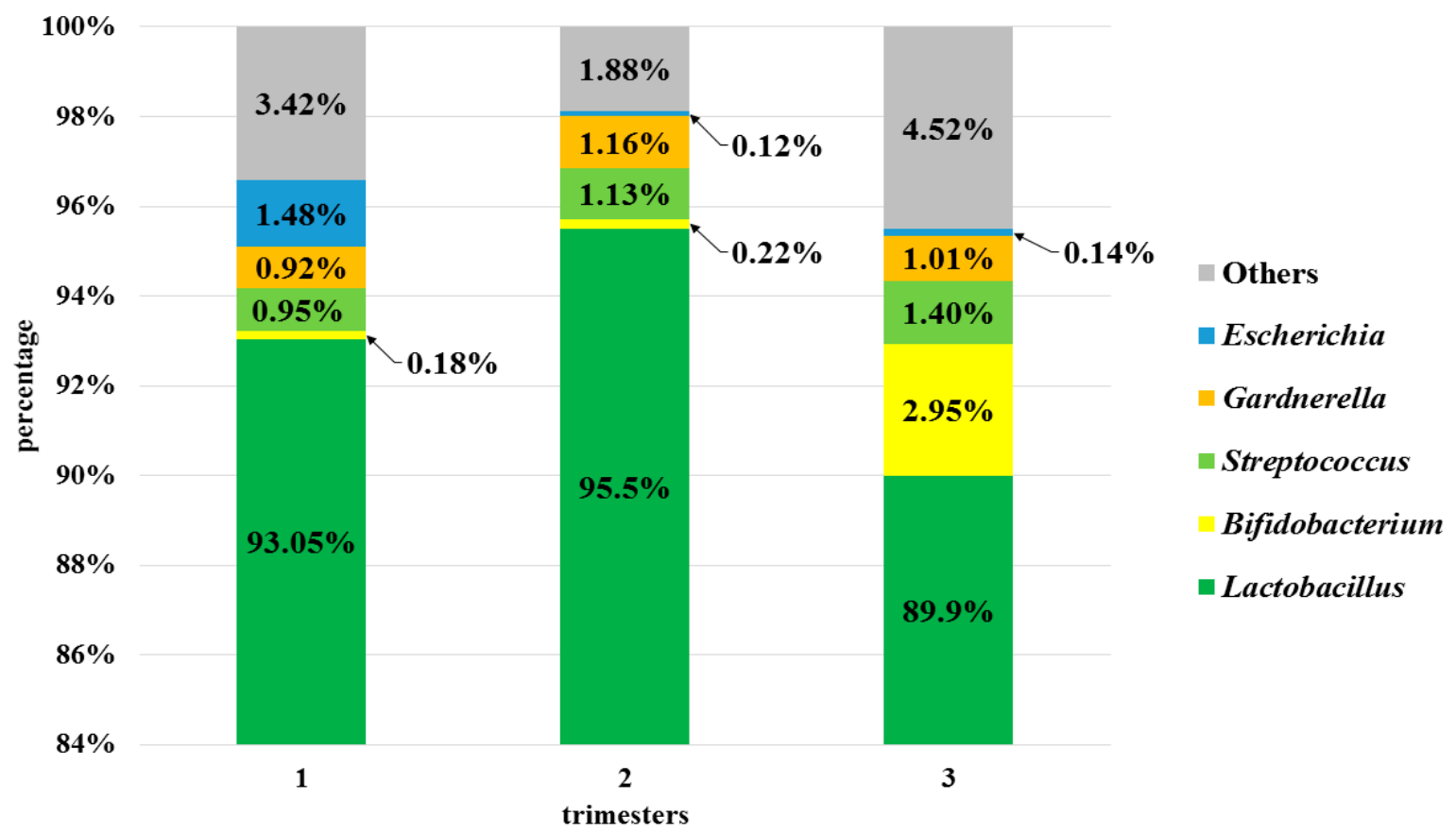

Figure 3. Bacterial profiles for 1st, 2nd and 3rd trimesters at the genus level (L6).

At level L7, 2535 species were identified, but each trimester in the studied group was dominated by species of the genus Lactobacillus: L. iners (52.26\%) L. gasseri (21.28\%), L. plantarum (9.14\%), L. acidophilus $(3.04 \%)$, and L. jensenii (3.00\%). Other species with a percentage greater than 1\% were: Gardnerella spp. (1.99\%), Streptococcus agalactiae (1.31\%), and Bifidobacterium breve (1.17\%) (Figure 4). 


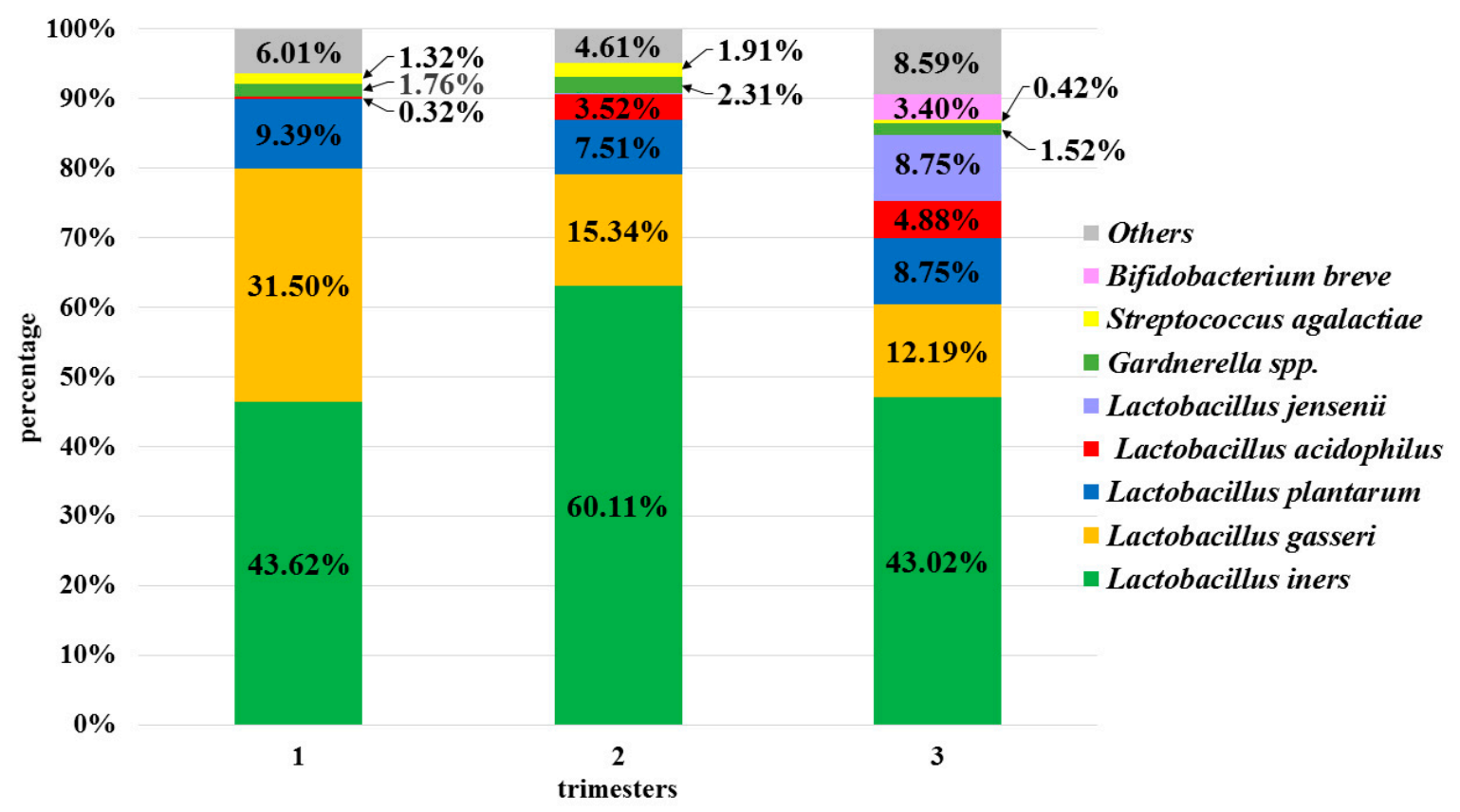

Figure 4. Bacterial profiles for 1st, 2nd and 3rd trimesters at the species level (L7).

The remaining species were present in negligible amounts. Statistically significant differences were observed between the 1st and 2nd trimesters with regard to the amounts of S. agalactiae and L. iners $(p<0.001)$, and between the 1st and 3rd with regard to the amounts of Gardnerella spp. $(p<0.001)$. There were also statistically significant differences in the abundance of $L$. jensenii over the three trimesters $(p<0.01)$.

\subsection{Semi-Quantitative and Qualitative Composition of the Physiological Microbiota in Individual Patients}

In the next part of the study, the vaginal microbiota from the three trimesters was analyzed individually for each patient. Based on the data obtained, the samples were divided into three categories:

\subsubsection{Stable Microbiota during Pregnancy}

Out of the 32 women, 12 (37.5\%) had a relatively stable composition of the vaginal microbiota during the whole period of pregnancy, and there were no statistically significant differences between species diversity and trimesters. Among these patients, $6(18.75 \%)$ were characterized by the domination of L. iners, which accounted for $60 \%$ to $99 \%$ of all species. In the remaining patients (43.75\%), other species of the genus Lactobacillus were in a similar percentage: L. gasseri (20-35\%), L. acidophilus (13-26\%), and L. helveticus (8-21\%).

3.4.2. Fluctuations in the Non-Pathogenic Potential Lactobacillus Species Composition of the Vaginal Microbiota

Bacterial profiles of $9(28.1 \%)$ more women showed the presence of species fluctuations during the pregnancy, mainly within the genus Lactobacillus. For example, in one patient, the number of L. jensenii in the 1st trimester was $95.7 \%$, while in subsequent trimesters, this species was replaced mainly by L. gasseri ( $87.1 \%$ in 2 nd trimester and $97.3 \%$ in 3rd trimester). In five patients $(15.63 \%)$, a high negative, but not significant, Spearman's correlation ( -0.976 to -0.997$)$ was observed between the amount of L. iners and L. gasseri. In three patients, an increase was observed in the percentage of L. iners over 3 trimesters (average percentage in the 1st trimester, $15.57 \% ; 82.1 \%$ in the 2nd and $75.05 \%$ in the 3rd) and a simultaneous decrease in the percentage of L. gasseri $(71.54 \%$ in the 1 st, $6.26 \%$ in the 2 nd and $1.25 \%$ in the $3 \mathrm{rd}$ ). The opposite relationship was observed in the other two patients: a decrease in the $L$. iners percentage $(69.7 \%$ in the 1 st, $12.75 \%$ in the 2 nd and $0.11 \%$ in the 3 rd) was 
accompanied by an increase in L. gasseri $(7.85 \%$ in the 1 st, $20.15 \%$ in the 2 nd, $23.71 \%$ in the the 3 rd trimester). However, probably due to the small number of observations, the determined correlations were statistically insignificant, but it is worth paying attention to in the context of future research. On the other hand, in the three remaining patients, similar relationships to those described above were noted in relation to L. iners and L. plantarum.

\subsubsection{Fluctuations in the Pathogenic Potential Species Composition of the Vaginal Microbiota}

In 11 women, a significant presence of vaginal microbiota with pathogenic potential was observed across the trimesters. The fluctuations were mainly related to a significant increase in Gardnerella spp., S. agalactiae, E. faecalis, and A. vaginae. Due to the large amount of data, detailed observations of bacterial profiles of only several patients are shown and described in Figure 5.

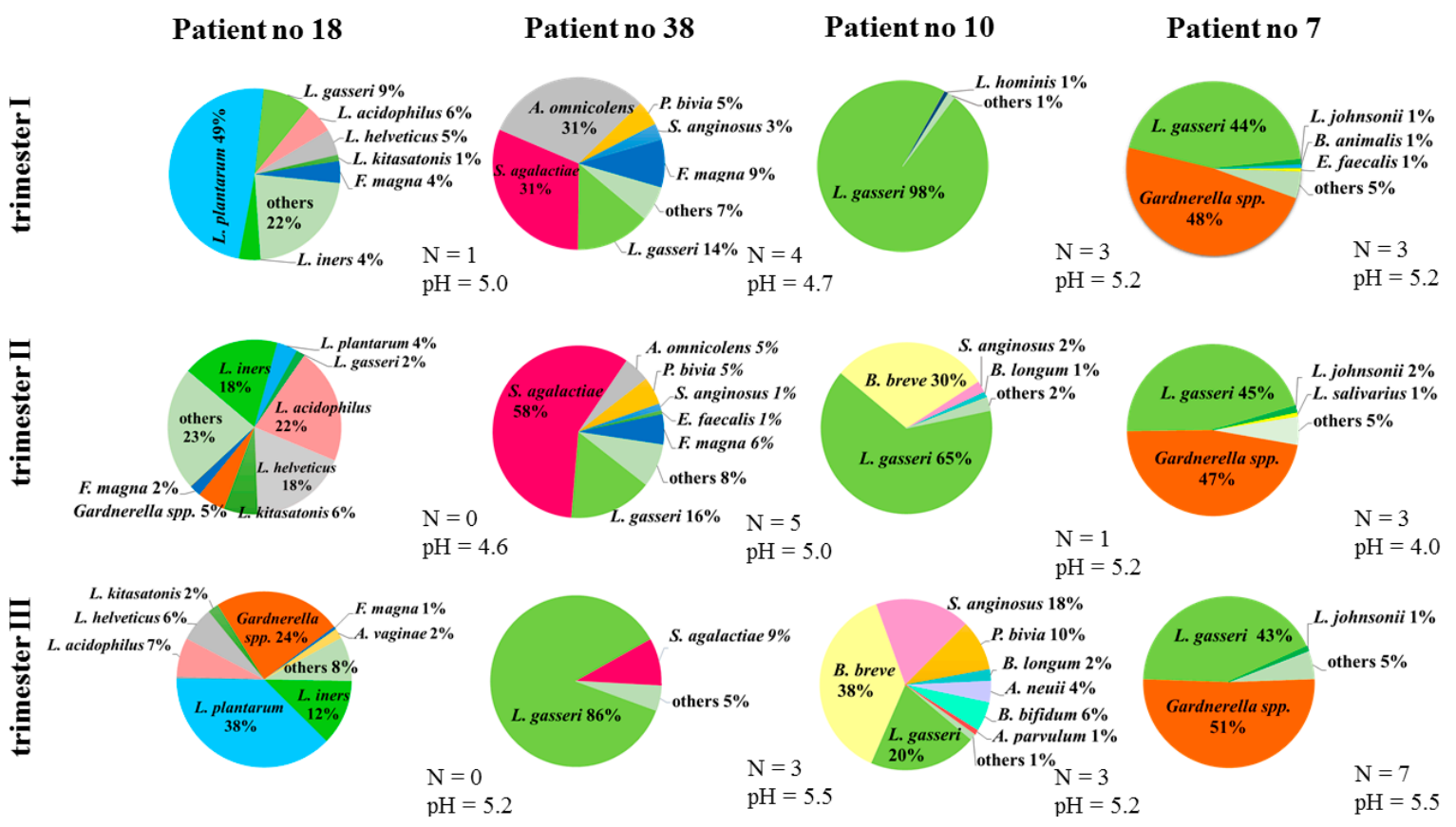

Figure 5. Bacterial profiles of selected patients, whose samples were clearly different from other pregnant women. For each sample and trimester, values of $\mathrm{pH}$ and Nugent score $(\mathrm{N})$ are provided under the pie chart.

During the first trimester of pregnancy, the vagina of patient number 18 was colonized mainly by L. plantarum and other species of the genus Lactobacillus. In the second trimester, this trend shifted to L. iners, L. acidophilus, and L. helveticus, and also, a small amount of Gardnerella spp. appeared. In the last trimester, the percentage of L. plantarum was restored, but significant amounts of Gardnerella spp. were also observed $(p<0.01)$. In patient number 38 , both in the first as well as the second trimesters, high percentages of $S$. agalactiae were noted $(p<0.01)$. In the third trimester, the bacterial balance was restored and the percentage of $S$. agalactiae was reduced to trace amounts. The next example of bacterial dynamics in the vagina is patient number 10. In the first trimester, her vaginal microbiota was homogeneous, dominated by L. gasseri. In the second trimester, B. breve and S. anginosus additionally appeared, but in the third trimester, $p$. bivia and $S$. anginosus reached statistically significant amounts compared to earlier trimesters $(p<0.01)$. Moreover, the amounts of L. gasseri were reduced almost five times $(p<0.01)$. Although in patient number 7 , it is difficult to reach conclusions about the dynamics, the bacterial profile was distinctly different from other patients. In all three trimesters, a considerable and significant percentage ( $p=0.03$ ) of Gardnerella spp. was present.

Patient number 6: During the 1st trimester, the vagina of the patient was colonised mainly by Enterococcus faecalis and Staphylococcus saprophyticus $(p<0.01)$. In the 2 nd and 3rd trimester these 
bacteria were observed in trace amounts. In relation to E. faecalis, a similar result was observed in another patient with pathogenic potential fluctuations.

Patient number 9: in the 1st trimester, low amounts of Gardnerella spp. and high amounts of GBS were observed, but in the 2nd and 3rd trimesters a reverse observation (also significant, $p<0.03$ ) was noted. In relation to GBS, similar results were observed in 2 other patients.

Patient number 16: in the 1st trimester, low amounts of Gardenrella spp. and Atopobium vaginae were noted, but these values in the 2nd and 3rd trimesters (both in relations to Gardnerella spp. and only in the 3rd in relations to Atopobium vaginae) were significantly higher $(p<0.01)$.

Patient number 42: a high percent of Lactobacillus gasseri was observed in the 1st and 2nd trimesters, but in 3rd, a significant decrease in the amounts of L. gasseri species. In the 3rd trimester, considerable and statistically significant percent of GBS appeared $(p<0.001)$.

\section{Discussion}

In our study, we demonstrated the semi-quantitative and qualitative composition of the vaginal microbiota during pregnancy in healthy Caucasian women, with the use of the next-generation sequencing method. The main limitation of the study was the small study cohort, therefore, the presented results require confirmation in the future in a larger research group. The strength of our research was the analysis of the bacterial composition at three-time points for one ethnic group and also for each patient individually. Until now, only a few works in the available literature have obtained samples from two or more trimesters and used the sequencing method [26-30]. In the study by Romero et al., although samples were taken, on average, six times during the whole pregnancy, the results were mainly related to African Americans $(n=19)$. Other ethnic groups that were isolated were White $(n=2)$ and Hispanic $(n=1)$ [26]. It was similar in another work by the same authors, where out of 72 women who delivered at term, only four were White. The vaginal microbiota of 12 pregnant women at four-time points (8-12, 17-21, 27-31, and 36-38 weeks of gestation) were also subject to the research of the team headed by Walther-Antonio et al. [30]. In the work of these authors, the attention was paid to differences in the bacterial vaginal composition between Caucasian and African-American pregnant women in relation to the findings of Romero et al. [30].

Our study results are in accordance with previously published works, in which it is emphasized that, during pregnancy, the physiological vaginal microbiota is dominated by the genus Lactobacillus $[10,26-29,31]$. The dominance of this genus in gestating women is associated with increased production of estrogen. As a consequence, glycogen is accumulated and metabolized to lactic acid, which promotes the proliferation of lactobacilli $[9,26,32]$.

It is worth noting that among the genus Lactobacillus, not all species are equal. The deficiency of L. iners and or L. gasseri may lead to greater susceptibility to pathogenic changes of the microbiota. However, this is not a rule. In our work, in relation to the analyzed group, L. iners was predominant among this genus, while others (L. acidophilus, L. helveticus, and L. gasseri) occurred in smaller amounts. In a systematic review, van de Wijgert et al. observed that, in the majority of studies, one cluster was dominant, i.e., L. crispatus (11 articles) or L. iners (15 articles), other species are less common [3] - data from 2014. Some authors have observed that L. iners prevails more often in Black African and African American women and also is connected with greater bacterial diversity compared to Caucasian or Asian women. In the latter group, L. crispatus, L. jensenii, L. gasseri, and/or L. vaginalis are predominant [26]. Walther-Antonio et al. suggest that the dominance of L. crispatus and L. iners is dependent on the pregnant woman's age, and L. iners is dominant in older gravidae (34-36 years old). Unfortunately, these observations are based on two units only [30]. The role of these species in the vagina during pregnancy is still unclear, but several articles suggest that $L$. iners can be dominant at the time when the microbiota is in the transition phase between "normal" and "abnormal" [33] or helps to restore the domination of Lactobacilli after antibiotic treatment [3]. This is also likely due to the fact that the L. iners genome also encodes inerolysin, a pore-forming toxin (related to Gardnerella spp. vaginolysin), 
and has clonal variants that promote vaginal health in some cases and are associated with dysbiosis and disease in others [34].

Another work suggests that L. iners is the only species of the genus Lactobacillus, which after using antibiotics, can dominate the bacterial microbiota of the vagina. Consequently, it can make patients more susceptible to new episodes of bacterial vaginal infections [35]. Moreover, it is associated with a higher prevalence of sexually transmitted infections [36].

In our research, only a small percentage of L. crispatus (from 0.53 to $0.58 \%$ ) was identified in relation to the above-mentioned ones. These results are surprising, especially when combined with previously published analyses carried out on the same experimental group but using Sanger sequencing [37]. In the previous study, the dominant species was L. crispatus (29\% in the 1 st trimester, $51.6 \%$ in the 2nd and $25.8 \%$ in the 3rd trimester); the second most frequent species was L. gasseri $(19.4 \%, 25.8 \%$, and $25.8 \%$, respectively) and then L. rhamnosus, L. amylovorus, and L. johnsonii. L. iners was not identified. Significant discrepancies between the results of the studies were obtained, which mainly stemmed from preparing the material from patients differently. In previous studies, the isolation of bacterial DNA was carried out on the basis of colonies grown only on solid MRS medium (Difco), and in this study, DNA was isolated from swabs suspended in physiological saline. The species L. iners is difficult to culture on MRS medium and requires the components contained in the blood medium for growth. As a result, it was not possible to observe the growth of this species and its identification. These studies show the limitations of culture and the advantages of molecular methods, but the latter are not without their drawbacks, which is discussed later on in the discussion.

The conducted studies showed that, during pregnancy, there are changes in the qualitative and semi-quantitative composition of bacteria in the vagina over the course of 3 trimesters at the genus and species level. Although in 12 out of 32 patients, these changes were not statistically significant, in the remaining patients, significant fluctuations in numbers and qualities, physiological or pathogenic in nature, were found. An interesting observation was to show a strong but insignificant (probably due to the small number of observations) negative correlation between L. iners and L. gasseri in the group of patients with physiological fluctuations. So far, no similar dependencies have been found in the available literature. These two species are present both in healthy pregnant women and those with dysbiosis [35] and are claimed to have a low impact on the stability of the microbiota. Moreover, in the work of Verstraelen et al., L. iners and L. gasseri are also mentioned as species that, with time, may strongly predispose to bacterial overgrowth in the vagina during pregnancy [35]. The above statements may justify the following successive results of our studies regarding patients in whom qualitative and/or semi-quantitative vaginal microbiota with pathogenic potential fluctuations have been observed during different periods of pregnancy. The samples in which the dominant species of the genus Lactobacillus was L. gasseri were characterized by a much greater diversity and percentage share of other bacteria, e.g., Gardnerella spp., S. agalactiae, Streptococcus anginosus, and Prevotella bivia.

Unfortunately, the exact role of L. gasseri is still unknown. Interesting results were published in the work by Atassi et al., where it was proved on the basis of a series of in-vitro experiments that some L. gasseri strains at $\mathrm{pH}>4$ show a bactericidal effect against $S$. agalactiae [38]. These conclusions may explain the pathogenic fluctuations observed in this publication in patient no 38 (Figure 5). Although a significant percentage of GBS ( $31 \%$ and $58 \%$, respectively) and a relatively low L. gasseri ( $14 \%$ and $16 \%$ ) were recorded in the first and second trimesters, these values reversed in the third trimester in favor of the species L. gasseri $(86 \%)$, unrivaled in the domination of the vaginal microbiota.

In the remaining patients with pathogenic fluctuations, in whom significant percentages of Lactobacillus species other than L. gasseri were found, typical bacteria described in the course of bacterial vaginosis (BV) were also identified: Gardnerella spp. and Atopobium vaginale. It should be noted here that none of the examined patients showed symptoms typical of BV. Although in the case of Gardnerella spp., the literature data indicate that, in as many as $80 \%$ of women, colonization with this bacterium is asymptomatic [38]. Moreover, on the basis of the research of Wong et al., the high incidence of Gardnerella spp. in women without confirmed BV does not refute the hypothesis that Gardnerella spp. 
is the causative factor for BV. The functional role of Gardnerella spp. in the vagina can vary greatly depending on the strains of these bacteria in health or illness $[39,40]$. On the other hand, the increased abundance of Gardnerella spp. leads to the formation of a proteolytic enzyme-rich biofilm on the surface of epithelial cells, which causes epithelial exfoliation, creating colonization sites for other anaerobes or facultative anaerobes, e.g., Atopobium vaginae. Further changes in the vaginal ecosystem characteristic for BV may predispose to infections with other microorganisms, e.g., Trichomonas vaginalis, Neisseria gonorrhoeae, and Chlamydia trachomatis [41].

In some cases, in our study, the abundance of bacteria of the genus Bifidobacterium (B. breve, B. longum and B. bifidum) was also found, especially in the 3rd trimester. It is probably an effect of progesterone, the principal gestational hormone, which reaches its highest concentrations in the last period of pregnancy and promotes an increase in the relative abundance of Bifidobacterium. Research by the team of Nuriel-Ohayon et al. in pregnant women, and also in a mouse model and under in vitro conditions, confirms these findings [42]. Species of the genus Bifidobacterium play an important role, not only in the pregnant mother (boosting the immune system, improving insulin sensitivity) but first of all in the infant. They are passed on to the newborn during natural birth and during breastfeeding and are the major type in the healthy infant's digestive tract [43]. Bifidobacteria play an important role in the maturation of the immune system early in life and produce lactic acid, which has the ability to metabolize human milk oligosaccharides. Reduced numbers of these bacteria in infants are associated with disease states [44]. Therefore, it can be assumed that the increase in the number of bacteria of the genus Bifidobacterium in the third trimester of pregnancy is associated with natural preparation for childbirth.

While Bifidobacterium is considered to be a component of the natural bacterial microbiota, Streptococcus and Prevotella are often found in bacterial disorders or bacterial vaginosis (BV) [45]. However, their presence should not always be associated with a pathogenic condition or infection (as above for Gardnerella spp.), because not every deviation from the normal composition of the vaginal bacterial microbiota indicates disease. For its development, the interaction between virulence factors and their quantitative domination is necessary. Accordingly, it can be argued that some taxa, depending on certain factors, may act as commensals or pathogens [46]. Moreover, some bacteria, which are also present in BV, e.g., Megasphaera or Atopobium, have the ability to produce lactic acid, which would indicate that it is possible for species other than lactobacilli to protect one against the proliferation of pathogenic bacteria [32]. For this reason, it is necessary to be able to properly interpret these data.

In the next-generation sequencing research, apart from the fact that interpretation of the results is often complicated, the methodology is also an important issue. First of all, the selection of suitable primer pairs and hypervariable regions of the bacterial 16S rRNA gene is significant and may have influence on obtaining different results. In our study, universal primers from the Illumina protocol were used, covering regions V3-V4, similarly to the research of Borgdorff et al. [36]. In the literature, we found information stating that for both short and longer read sequences, region V4, which is also included in our set of primers, is appropriate for capturing microbial diversity [5]. The results of our research showed that, with the use of these primer pairs, it is possible to reliably identify all groups of bacteria which are reported in similar studies. However, it would be necessary to perform comparative studies in the future using primers amplifying other hypervariable regions of the bacterial 16S rRNA gene. The problem of choosing the proper primer pairs in research on the vaginal microbiota is quite a widely discussed issue. Aagard, DiGiulio and Walther-Antonio and co-workers, in their research, used primers covering regions V3-V5 [27,29,30], Subramaniam et al.: V4 [5], Fettweis and Virtanen with co-workers: V1-V3 [6,47], MacIntyre and Romero and co-workers: V1-V2 [10,26]. Although each of these regions will allow insight into the bacterial community in the sample, it should be noted that the results will be flawed. For instance, the set of primers V1-V3 makes it possible to distinguish between Lactobacillus species but may underestimate the genera Acinetobacter and Escherichia. Moreover, it does not fully distinguish the family Enterobacteriaceae and some other genera, for example, Staphylococcus. Other sets of primers, V3-V5, are better for the study of Enterobacteriaceae and Bifidobacteriaceae but 
not suited to identify the species from Lactobacillus and Prevotella genus [9]. Similarly, the set of primers V1-V2 allows to identify some phylotypes at the genus level, but fewer at the species level [26]. Until now, no optimal solution has been found, so further research is needed to select the right, universal primers to study the vaginal microbiota.

Another important methodological issue is the selection of tools and databases for comparing and assigning sequences to the appropriate taxonomic levels with the increasing emphasis on species-level classification. The most commonly used tools are: RDP Classifier [48], 16S Classifier [49], Kraken classifier, GOTTCHA, LMAT, OneCodex [50] SPINGO, SILVA [51], and Greengenes [52], software with the classification option, e.g., QIIME [53] and MG-RAST [54]. Despite the large selection and general availability of these tools, classification at the species level still presents problems, which are widely discussed, among others, in the works of Gao et al. [54] and Tuzhikov et al. [55]. A comparative study conducted by Lindgreen et al. showed that none of these classifiers could be used as the best choice for sequence analysis [56]. In our research, there was only $47-57 \%$ sequence alignment at the species level, which is a much lower percentage compared to the higher taxonomic levels (Table 3).

Next-generation sequencing requires further improvement and unification of some tools that allow proper determination of the vaginal taxonomic composition, primarily the development of universal primers and the creation of one reference database. Despite these limitations, NGS is currently the quickest and most accurate solution in vaginal microbiota testing compared to culture methods. Moreover, it can also be useful in difficult cases when multiple bacterial agents are involved.

\section{Conclusions}

In our study, we have shown that the vaginal microbiota in the group of pregnant women is subject to partial changes during the three trimesters of pregnancy. Although these changes were not reflected in the values of the alpha and beta diversity parameters, they were significant, especially at the genus and species level. In the group of patients with physiological fluctuations, they mainly concerned Lactobacillus iners, and in patients with pathogenic fluctuations, Streptococcus agalactiae and Gardnerella spp. The most abundant genus Lactobacillus was represented mainly by Lactobacillus iners. Moreover, an interesting trend was observed, which concerned a strong negative but insignificant correlation between the abundance of L. iners and L. gasseri. It is worth paying attention to this relationship in the context of future research.

Author Contributions: Conceptualization, M.B.-W. and T.G.; methodology, A.S.-O. and T.G. software, A.G.; validation, T.G., formal analysis, A.G.; T.G., and M.B.-W., investigation, A.S.-O.; resources, W.P., M.B.-W., P.K., A.H.L.-S., and P.P.W.; data curation, A.S.-O. and A.G.; writing-original draft preparation, A.S.-O.; writing-review and editing, M.B.-W., T.G., A.G., A.H.L.-S., P.K., and P.P.W.; visualization, A.S.-O. and A.G.; supervision, M.B.-W.; project administration, M.B.-W.; funding acquisition, M.B.-W. All authors have read and agreed to the published version of the manuscript.

Funding: This research was partially funded by grant number TANGO2/340018/NCBR/2017 financed by the National Science Center and the National Center for Research and Development. Research funding from the TANGO2 project included the development and standardization of a new GBS diagnostic methods for carrier and infection in relation to the classical method of culture and other molecular methods. The samples analyzed in this work were derived from the previous studies, because we wanted to develop the method on a complete set of samples and fully confirm its effectiveness.

Conflicts of Interest: The authors declare no conflict of interest. The funders had no role in the design of the study; in the collection, analyses, or interpretation of data; in the writing of the manuscript, or in the decision to publish the results.

\section{References}

1. Hickey, R.J.; Zhou, X.; Pierson, J.D.; Ravel, J.; Forney, L.J. Understanding vaginal microbiome complexity from an ecological perspective. Transl. Res. 2012, 160, 267-282. [CrossRef] [PubMed]

2. Ma, B.; Forney, L.J.; Ravel, J. Vaginal Microbiome: Rethinking Health and Disease. Annu. Rev. Microbiol. 2012, 66, 371-389. [CrossRef] [PubMed] 
3. Van De Wijgert, J.; Borgdorff, H.; Verhelst, R.; Crucitti, T.; Francis, S.; Verstraelen, H.; Jespers, V. The Vaginal Microbiota: What Have We Learned after a Decade of Molecular Characterization? PLoS ONE 2014, 9, e105998. [CrossRef] [PubMed]

4. Nuriel-Ohayon, M.; Neuman, H.; Koren, O. Microbial Changes during Pregnancy, Birth, and Infancy. Front. Microbiol. 2016, 7, 1031. [CrossRef]

5. Kumar, R.; Cliver, S.P.; Zhi, D.; Szychowski, J.M.; Abramovici, A.; Biggio, J.R.; Lefkowitz, E.J.; Morrow, C.; Edwards, R.K.; Subramaniam, A. Vaginal Microbiota in Pregnancy: Evaluation Based on Vaginal Flora, Birth Outcome, and Race. Am. J. Perinatol. 2016, 33, 401-408. [CrossRef]

6. Fettweis, J.M.; Serrano, M.G.; Girerd, P.H.; Jefferson, K.K.; Buck, G.A. A New Era of the Vaginal Microbiome: Advances Using Next-Generation Sequencing. Chem. Biodivers. 2012, 9, 965-976. [CrossRef]

7. Dunlop, A.L.; Mulle, J.G.; Ferranti, E.P.; Edwards, S.; Dunn, A.B.; Corwin, E.J. Maternal Microbiome and Pregnancy Outcomes That Impact Infant Health: A Review. Adv. Neonatal Care 2015, 15, 377-385. [CrossRef]

8. Ravel, J.; Brotman, R.M. Translating the vaginal microbiome: Gaps and challenges. Genome Med. 2016, 8, 35. [CrossRef]

9. Prince, A.L.; Chu, D.M.; Seferovic, M.D.; Antony, K.M.; Ma, J.; Aagaard, K.M. The Perinatal Microbiome and Pregnancy: Moving Beyond the Vaginal Microbiome. Cold Spring Harb. Perspect. Med. 2015, 5, a023051. [CrossRef]

10. MacIntyre, D.A.; Chandiramani, M.; Lee, Y.S.; Kindinger, L.; Smith, A.; Angelopoulos, N.; Lehne, B.; Arulkumaran, S.; Brown, R.; Teoh, T.G.; et al. The vaginal microbiome during pregnancy and the postpartum period in a European population. Sci. Rep. 2015, 5, 8988. [CrossRef]

11. Brzychczy-Włoch, M.; Ochońska, D.; Bulanda, M. Carriage of Group B Streptococci in Pregnant Women from The Region of Krakow and their Antibiotic Resistance in the Years 2008-2012. Pol. J. Microbiol. 2013, 62, 427-433. [CrossRef] [PubMed]

12. Eloe-Fadrosh, E.A.; Rasko, D.A. The Human Microbiome: From Symbiosis to Pathogenesis. Annu. Rev. Med. 2013, 64, 145-163. [CrossRef] [PubMed]

13. Haque, M.M.; Merchant, M.; Kumar, P.N.; Dutta, A.; Mande, S.S. First-trimester vaginal microbiome diversity: A potential indicator of preterm delivery risk. Sci. Rep. 2017, 7, 1-10. [CrossRef] [PubMed]

14. Bagga, R.; Arora, P. Genital micro-organisms in pregnancy. Front. Public Health 2020, 8, 225. [CrossRef]

15. Brzychczy-Wloch, M.; Pabian, W.; Majewska, E.; Zuk, M.G.; Kielbik, J.; Gosiewski, T.; Bulanda, M.G. Dynamics of colonization with group B streptococci in relation to normal flora in women during subsequent trimesters of pregnancy. New Microbiol. 2014, 37, 307-319.

16. Nugent, R.P.; Krohn, M.A.; Hillier, S.L. Reliability of diagnosing bacterial vaginosis is improved by a standardized method of gram stain interpretation. J. Clin. Microbiol. 1991, 29, 297-301. [CrossRef]

17. Gosiewski, T.; Szała, L.; Pietrzyk, A.; Brzychczy-Włoch, M.; Heczko, P.B.; Bulanda, M. Comparison of Methods for Isolation of Bacterial and Fungal DNA from Human Blood. Curr. Microbiol. 2014, 68, 149-155. [CrossRef]

18. 16S Metagenomic Sequencing Library Preparation; Preparing 16S Ribosomal RNA Gene Amplicons for the Illumina MiSeq System. Available online: https://Support.illumina.com (accessed on 14 October 2020).

19. Wang, Q.; Garrity, G.M.; Tiedje, J.M.; Cole, J.R. Native Bayesian Classifier for Rapid Assignment of rRNA Sequences into the New Bacterial Taxonomy. Appl. Environ. Microbiol. 2007, 73, 5261-5267. [CrossRef]

20. Edgar, R.C.; Haas, B.J.; Clemente, J.C.; Quince, C.; Knight, R. UCHIME improves sensitivity and speed of chimera detection. Bioinformatics 2011, 27, 2194-2200. [CrossRef]

21. Alishum, A. DADA2 formatted 16S rRNA gene sequences for both bacteria and archaea. Res. Data 2019.

22. Chong, J.; Liu, P.; Zhou, G.; Xia, J. Using Microbiome Analyst for comprehensive statistical, functional, and meta-analysis of microbiome data. Nat. Protoc. 2020, 15, 799-821. [CrossRef] [PubMed]

23. Love, M.I.; Huber, W.; Anders, S. Moderated estimation of fold change and dispersion for RNA-seq data with DESeq2. Genome Biol. 2014, 15, 550. [CrossRef] [PubMed]

24. Yoav, B.; Hochberg, Y. Controlling the False Discovery Rate: A Practical and Powerful Approach to Multiple Testing. J. R. Stat. Soc. Ser. B 1995, 57, 289-300.

25. JASP; Version 0.14; Computer Software; JASP Team: Amsterdam, The Netherlands, 2020.

26. Romero, R.; Hassan, S.S.; Gajer, P.; Tarca, A.L.; Fadrosh, D.W.; Nikita, L.; Galuppi, M.; Lamont, R.F.; Chaemsaithong, P.; Miranda, J.; et al. The composition and stability of the vaginal microbiota of normal pregnant women is different from that of non-pregnant women. Microbiome 2014, 2, 4. [CrossRef] 
27. DiGiulio, D.B.; Callahan, B.J.; McMurdie, P.J.; Costello, E.K.; Lyell, D.J.; Robaczewska, A.; Sun, C.L.; Goltsman, D.S.A.; Wong, R.J.; Shaw, G.; et al. Temporal and spatial variation of the human microbiota during pregnancy. Proc. Natl. Acad. Sci. USA 2015, 112, 11060-11065. [CrossRef]

28. Hyman, R.W.; Bs, M.F.; Jiang, H.; Fung, E.; Rand, L.; Bs, B.J.; Vo, K.C.; Caughey, A.B.; Hilton, J.F.; Davis, R.W.; et al. Diversity of the vaginal microbiome correlates with preterm birth. Reprod. Sci. 2014, 21, 32-40. [CrossRef]

29. Aagaard, K.M.; Riehle, K.; Ma, J.; Segata, N.; Mistretta, T.-A.; Coarfa, C.; Raza, S.; Rosenbaum, S.; Veyver, I.V.D.; Milosavljevic, A.; et al. A Metagenomic Approach to Characterization of the Vaginal Microbiome Signature in Pregnancy. PLoS ONE 2012, 7, e36466. [CrossRef]

30. Walther-António, M.R.S.; Jeraldo, P.; Miller, M.E.B.; Yeoman, C.J.; Nelson, K.E.; Wilson, B.A.; White, B.A.; Chia, N.; Creedon, D.J. Pregnancy's Stronghold on the Vaginal Microbiome. PLoS ONE 2014, 9, e98514. [CrossRef]

31. Hernández-Rodríguez, C.; Romero-González, R.; Albani-Campanario, M.; Figueroa-Damián, R.; Meraz-Cruz, N.; Hernández-Guerrero, C. Vaginal microbiota of healthy pregnant mexican women is constituted by four Lactobacillus species and several vaginosis-associated bacteria. Infect. Dis. Obstet. Gynecol. 2011, 2011, 1-9. [CrossRef]

32. Linhares, I.M.; Kanninen, T.T.; Orfanelli, T.; Jayaram, A.; Doulaveris, G.; Witkin, S.S. The Vaginal Microbiome: New Findings Bring New Opportunities: The Vaginal Microbiome. Drug Dev. Res. 2013, 74, 360-364. [CrossRef]

33. Jakobsson, T.; Forsum, U. Lactobacillus iners: A marker of changes in the vaginal flora? J. Clin. Microbiol. 2007, 45, 3145. [CrossRef] [PubMed]

34. Petrova, M.I.; Reid, G.; Vaneechoutte, M.; Lebeer, S. Lactobacillus iners: Friend or foe? Trends Microbiol. 2017, 25, 182-191. [CrossRef] [PubMed]

35. Verstraelen, H.; Verhelst, R.; Claeys, G.; De Backer, E.; Temmerman, M.; Vaneechoutte, M. Longitudinal analysis of the vaginal microflora in pregnancy suggests that L. crispatus promotes the stability of the normal vaginal microflora and that L. gasseri and/or L. iners are more conducive to the occurrence of abnormal vaginal microflora. BMC Microbiol. 2009, 9, 116. [CrossRef] [PubMed]

36. Borgdorff, H.; Van Der Veer, C.; Van Houdt, R.; Alberts, C.J.; De Vries, H.J.; Bruisten, S.M.; Snijder, M.B.; Prins, M.; Geerlings, S.E.; Van Der Loeff, M.F.S.; et al. The association between ethnicity and vaginal microbiota composition in Amsterdam, the Netherlands. PLoS ONE 2017, 12, e0181135. [CrossRef]

37. Dobrut, A.; Gosiewski, T.; Pabian, W.; Bodaszewska-Lubaś, M.; Ochońska, D.; Bulanda, M.; Brzychczy-Włoch, M. The dynamics of vaginal and rectal Lactobacillus spp. flora in subsequent trimesters of pregnancy in healthy Polish women, assessed using the Sanger sequencing method. BMC Pregnancy Childbirth 2018, 18, 350. [CrossRef]

38. Atassi, F.; Brassart, D.; Grob, P.; Graf, F.; Servin, A.L. Lactobacillus strains isolated from the vaginal microbiota of healthy women inhibit Prevotella bivia and Gardnerella vaginalis in coculture and cell culture. FEMS Immunol. Med Microbiol. 2006, 48, 424-432. [CrossRef]

39. Wong, Y.P.; Tan, G.C.; Wong, K.K.; Anushia, S.; Cheah, F.C. Gardnerella vaginalis in perinatology: An overview of the clinicopathological correlation. Malays. J. Pathol. 2018, 40, 267-286.

40. Janulaiiene, M.; Paliulye, V.; Pleckaityte, M. Prevalence and distribution of Gardnerella vaginalis subgroups in women with and without bacterial vaginosis. BMC Infect. Dis. 2017, 17, 394.

41. Recommendation of the Polish Society of Gynecologists and Obstetricians Regarding the Use of Antiseptics in Cases Non-Specific Vaginitis. 2020. Available online: http://ptgin.pl/ (accessed on 12 November 2020).

42. Nuriel-Ohayon, M.; Neuman, H.; Ziv, O.; Belogolovski, A.; Barsheshet, Y.; Bloch, N.; Uzan, A.; Lahav, R.; Peretz, A.; Frishman, S.; et al. Progesterone increases Bifidobacterium relative abundance during late pregnancy. Cell Rep. 2019, 27, 730-736. [CrossRef]

43. Ruiz, L.; Delgado, S.; Ruas-Madiedo, P.; Sanchez, B.; Margolles, A. Bifidobacteria and Their Molecular Communication with the Immune System. Front. Microbiol. 2017, 8, 2345. [CrossRef]

44. Peirotén, A.; Arqués, J.L.; Medina, M.; Rodríguez Mínguez, E. Bifidobacterial strains shared by mother and child as source of probiotics. Benef. Microbes 2018, 9, 231-238. [CrossRef] [PubMed]

45. Goltsman, D.S.A.; Sun, C.L.; Relman, D.; Proctor, D.M.; DiGiulio, D.B.; Robaczewska, A.; Thomas, B.C.; Shaw, G.M.; Stevenson, D.K.; Holmes, S.P.; et al. Metagenomic analysis with strain-level resolution reveals fine-scale variation in human pregnancy microbiome. Genome Res. 2018, 28, 1467-1480. [CrossRef] [PubMed] 
46. Mendz, G.; Kaakoush, N.; Quinlivan, J. New techniques to characterise the vaginal microbiome in pregnancy. AIMS Microbiol. 2016, 2, 55-68. [CrossRef]

47. Virtanen, S.; Kalliala, I.; Nieminen, P.; Salonen, A. Comparative analysis of vaginal microbiota sampling using 16S rRNA gene analysis. PLoS ONE 2017, 12, e0181477. [CrossRef] [PubMed]

48. Cole, J.R.; Wang, Q.; Fish, J.A.; Chai, B.; McGarrell, D.M.; Sun, Y.; Brown, C.T.; Porras-Alfaro, A.; Kuske, C.R.; Tiedje, J.M. Ribosomal Database Project: Data and tools for high throughput rRNA analysis. Nucleic Acids Res. 2014, 42, 633-642. [CrossRef]

49. Chaudhary, N.; Sharma, A.K.; Agarwal, P.; Gupta, A.; Sharma, V.K. 16S Classifier: A Tool for Fast and Accurate Taxonomic Classification of 16S rRNA Hypervariable Regions in Metagenomic Datasets. PLoS ONE 2015, 10, e0116106. [CrossRef]

50. Martínez-Porchas, M.; Villalpando-Canchola, E.; Vargas-Albores, F. Significant loss of sensitivity and specificity in the taxonomic classification occurs when short 16S rRNA gene sequences are used. Heliyon 2016, 2, e00170. [CrossRef]

51. Quast, C.; Pruesse, E.; Yilmaz, P.; Gerken, J.; Schweer, T.; Yarza, P.; Peplies, J.; Glöckner, F.O. The SILVA ribosomal RNA gene database project: Improved data processing and web-based tools. Nucleic Acids Res. 2012, 41, D590-D596. [CrossRef]

52. DeSantis, T.Z.; Hugenholtz, P.; Larsen, N.; Rojas, M.; Brodie, E.L.; Keller, K.; Huber, T.; Dalevi, D.; Hu, P.; Andersen, G.L. Greengenes, a Chimera-Checked 16S rRNA Gene Database and Workbench Compatible with ARB. Appl. Environ. Microbiol. 2006, 72, 5069-5072. [CrossRef]

53. Caporaso, J.G.; Kuczynski, J.; Stombaugh, J.; Bittinger, K.; Bushman, F.D.; Costello, E.K.; Fierer, N.; Peña, A.G.; Goodrich, J.K.; Gordon, J.I.; et al. QIIME allows analysis of high-throughput community sequencing data. Nat. Methods 2010, 7, 335-336. [CrossRef]

54. Gao, X.; Lin, H.; Revanna, K.; Dong, Q. A Bayesian taxonomic classification method for 16S rRNA gene sequences with improved species-level accuracy. BMC Bioinform. 2017, 18, 247. [CrossRef] [PubMed]

55. Tuzhikov, A.; Panchin, A.; Shestopalov, V.I. TUIT, a BLAST-based tool for taxonomic classification of nucleotide sequences. BioTechniques 2014, 56, 78-84. [CrossRef] [PubMed]

56. Lindgreen, S.; Adair, K.L.; Gardner, P.P. An evaluation of the accuracy and speed of metagenome analysis tools. Sci. Rep. 2016, 6, 19233. [CrossRef] [PubMed]

Publisher's Note: MDPI stays neutral with regard to jurisdictional claims in published maps and institutional affiliations.

(C) 2020 by the authors. Licensee MDPI, Basel, Switzerland. This article is an open access article distributed under the terms and conditions of the Creative Commons Attribution (CC BY) license (http://creativecommons.org/licenses/by/4.0/). 